\title{
A deceptive step towards quantum speedup detection
}

\author{
Salvatore Mandrà ${ }^{1,2, *}$ and Helmut G. Katzgraber ${ }^{3,4,5, \dagger}$ \\ ${ }^{1}$ Quantum Artificial Intelligence Lab., NASA Ames Research Center, Moffett Field, CA 94035, USA \\ ${ }^{2}$ Stinger Ghaffarian Technologies Inc., 7701 Greenbelt Rd., Suite 400, Greenbelt, MD 20770 \\ ${ }^{3}$ Department of Physics and Astronomy, Texas A\&M University, College Station, Texas 77843-4242, USA \\ ${ }^{4} 1 Q B$ Information Technologies (1QBit), Vancouver, British Columbia, Canada V6B 4 W4 \\ ${ }^{5}$ Santa Fe Institute, 1399 Hyde Park Road, Santa Fe, New Mexico 87501 USA
}

(Dated: July 4, 2018)

\begin{abstract}
There have been multiple attempts to design synthetic benchmark problems with the goal of detecting quantum speedup in current quantum annealing machines. To date, classical heuristics have consistently outperformed quantum-annealing based approaches. Here we introduce a class of problems based on frustrated cluster loops — deceptive cluster loops — for which all currently known state-of-the-art classical heuristics are outperformed by the DW2000Q quantum annealing machine. While there is a sizable constant speedup over all known classical heuristics, a noticeable improvement in the scaling remains elusive. These results represent the first steps towards a detection of potential quantum speedup, albeit without a scaling improvement and for synthetic benchmark problems.
\end{abstract}

PACS numbers: 75.50.Lk, 75.40.Mg, 05.50.+q, 03.67.Lx

\section{INTRODUCTION}

Quantum annealing (QA) [1-6] has been proposed as a potentially efficient heuristic to optimize hard constraint satisfaction problems. In principle, the approach can overcome tall energy barriers commonly found in this class of optimization problems by exploiting quantum effects, thereby potentially outperforming commonly-used heuristics that use thermal kicks to overcome the barriers. However, despite a significant effort by the scientific community towards an optimization technique that, in principle, relies on quantum effects, it is still unclear whether quantum speedup is actually achievable using analog transverse-field quantum annealing approaches.

There have been multiple attempts to define quantum speedup [7, 8], as well as quantify any "quantumness" and problem-solving efficacy of current commercially-available quantum annealers [7, 9-22]. However, to date, any convincing detection of an improved scaling of quantum annealing with a transverse field over state-of-the-art classical optimization algorithms remains elusive. The increase in performance of quantum annealing machines in the last few years has resulted in an "arms race" with classical optimization algorithms implemented on CMOS hardware. The goal post to detect quantum speedup continuously keeps moving and has resulted in a renaissance in classical algorithm design to optimize hard constraint-satisfaction problems.

A key ingredient in the detection of quantum speedup is the selection of the optimization problems to be used as benchmark. Ideally, one would want a real-world industrial application where the time to solution of the quantum device scales better than any known algorithm

\footnotetext{
*salvatore.mandra@nasa.gov

† hgk@tamu.edu
}

with the size of the input. However, such application problems are not suitable for present-day quantum annealers, either because they require more variables than currently available or because precision requirements cannot be met by current technologies. Random spin-glass problems have been shown to be too easy to detect any scaling improvements $[12,13]$. As such, efforts have shifted to carefully-designed synthetic problems. While some studies focus on post-selection techniques [13], others focus on the use of planted solutions [15, 18, 21], or the use of gadgets [22]. Unfortunately, however, in all planted problems $[15,18,21]$ used to date, as well as problems that use gadgets [20], the underlying logical structure is easily decoded and the underlying problem trivially solved, sometimes even with exact polynomial methods [8]. Therefore, in the quest for quantum speedup, an important step is to design problems where no variable reduction or algorithmic trick can be exploited to reduce the complexity of the problem. Ideally, the benchmark problem should be hard for a small number of variables and "break" all known optimization heuristics.

In this work we introduce a class of benchmark problems designed for DW2000Q quantum annealers whose logical structure is not directly recognizable and whose typical computational complexity can be tuned via a control parameter that tunes the relative strength of inter- vs intracell couplers in the DW2000Q Chimera [23] topology. Note that this approach can be easily generalized to other topologies. We demonstrate that for a particular setting of the control parameter where the ground state of the virtual problem cannot be decoded, the D-Wave Systems Inc. DW2000Q quantum annealer outperforms all known classical optimization algorithms by approximately two to three orders of magnitude. More precisely, we compare against the two best heuristics to solve Ising-like problems on the DW2000Q Chimera topology, the Hamzede Freitas-Selby (HFS) [24, 25] and parallel tempering 
Monte Carlo with isoenergetic cluster moves $(\mathrm{PT}+\mathrm{ICM})$ [26] heuristics. Although we were not able to identify the optimal annealing time given the hard limit of $1 \mu \mathrm{s}$ as minimum annealing time in the DW2000Q device, the scaling is comparable and the speedup persists for increasing system sizes. Therefore, we present the first steps towards the detection of potential quantum speedup, however, for now, without a noticeable scaling improvement. Problems with tunable complexity as the ones shown here, combined with a careful statistical analysis, bulletproof definitions of quantum speedup, the inclusion of power consumption in the analysis, as well as the use of the currently best-available heuristics are key in the assessment of the performance of quantum-enhanced optimization techniques.

The paper is structured as follows. In Sec. II we present some technical details of this study. In particular, we introduce the benchmark problems used in Sec. III. Results are outlined in Sec. IV, followed by a discussion and concluding remarks.

\section{TECHNICAL DETAILS}

The DW2000Q quantum annealer is designed to optimize classical problem Hamiltonians of the quadratic form

$$
\mathcal{H}_{\mathrm{P}}=\sum_{(i, j) \in \mathcal{G}} J_{i j} \sigma_{i}^{z} \sigma_{j}^{z}+\sum_{i} h_{i} \sigma_{i}^{z}
$$

where $\mathcal{G}$ is known as Chimera graph [23] constructed of a two-dimensional lattice of fully-connected $\mathrm{K}_{4,4}$ cells. The couplers $\left\{J_{i j}\right\}$ and biases $\left\{h_{i}\right\}$ are programmable parameters that define the optimization problem to be studied. Although the DW2000Q Chimera architecture graph has been kept fixed since the first commercial generation of the machine, the number of qubits doubled almost every two years. At the moment, the latest DW2000Q chip counts 2023 working flux-qubits and 5871 working couplers. To minimize the cost function $H_{\mathrm{P}}$, the DW2000Q quantum chip anneals quantum fluctuations driven by a transverse field of the form

$$
\mathcal{H}_{\mathrm{D}}=\sum_{i} \sigma_{i}^{x}
$$

More precisely, the annealing protocol starts with the system initialized to a quantum paramagnetic state. Then, the amplitude of $H_{\mathrm{D}}$ is slowly reduced while the amplitude of the problem Hamiltonian $H_{\mathrm{P}}$ is gradually increased. If the annealing is slow enough, the adiabatic theorem [27] ensures that the quantum system remains in its instantaneous lowest energy state for the entire annealing protocol. Therefore, (close-to) optimal configurations for $H_{\mathrm{P}}$ can be retrieved by measuring the state of the qubits along the $z$-basis at the end of the anneal.

Given its intrinsic analog nature, combined with the heuristic properties of quantum annealing, the
DW2000Q device is only able to find the optimum of a cost function up to a probability $p$. Indeed, fast annealing in proximity of level crossings [1, 2, 28], as well as quantum dephasing effects $[10,29,30]$, thermal excitations [31-33] and programming errors [13, 34], can lead to higher energy states of $H_{\mathrm{P}}$ at the end of the anneal. A commonly-accepted metric is the time-to-solution (TTS). The TTS is defined as the time needed for a heuristic, either classical or quantum, to find the lowest energy state with $99 \%$ success probability, that is:

$$
\mathrm{TTS}=t_{\mathrm{run}} R_{99}=t_{\mathrm{run}} \cdot \frac{\log (0.01)}{\log (1-p)},
$$

where $t_{\text {run }}$ is either the running time (for a classical heuristic) or the annealing time (for the DW2000Q quantum chip) and $R_{99}$ is the number of repetitions needed to reach the desired success probability [7]. In this work we analyze the TTS as a function of the number of input variables in the problem.

\section{SYNTHETIC BENCHMARK PROBLEMS}

In this Section we outline and discuss a new synthetic benchmark we call "deceptive cluster loop" (DCL) problems based on traditional frustrated cluster loop problems. However, DCL problems have a tunable parameter that for particular values hides the underlying logical structure of the planted problem, thus "deceptive."

\section{A. Traditional frustrated cluster loop problems}

Based on the fact that it is typically hard for agnostic optimization algorithms to find the lowest energy state of very long frustrated chains, the frustrated cluster loop à la Hen (H-FCL) is a random model that has been proven to be hard for many classical heuristics [15]. The idea is simple: Given $\mathcal{G}$, an arbitrary connectivity graph for the problem Hamiltonian $\mathcal{H}_{\mathrm{P}}$, and two parameters $\alpha$ and $R$, H-FCL instances are constructed as follows:

1. Generate $M=\alpha n$ loops on the graph, where $n$ is the number of nodes in $\mathcal{G}$. Loops are constructed by placing random walkers on random nodes (tails are eliminated once random walkers cross their own path).

2. For each loop $k$, assign $J_{i j}^{k}=-1$ to all the corresponding couplings but one randomly chosen one, for which the value $J_{i j}^{k}=+1$ is assigned instead.

3. The final Hamiltonian is then constructed by adding up all the loop couplings, i.e.,

$$
H_{P}=\sum_{(i, j) \in \mathcal{G}} \sum_{k=1}^{M} J_{i j}^{k} \sigma_{i}^{z} \sigma_{j}^{z} .
$$


The instance is discarded if there is a coupling such that

$$
\left|\sum_{k=1}^{M} J_{i j}^{k}\right|>R .
$$

The parameters $\alpha$ and $R$ correspond to the density of "constraints" and to the "ruggedness" of the H-FCL problem, respectively.

Although the H-FCL problems can be, in principle, directly generated for the Chimera graph [15], in a recent paper [21], King et al. have chosen a different approach (called here K-FCL) that can be divided into two steps:

1. All couplings inside a $\mathrm{K}_{4,4}$ unit cell of the Chimera structure are set to be ferromagnetic, i.e., $J_{i j}=-1$, $\forall i, j \in \mathrm{K}_{4,4}$. Because the unit cells are fullyconnected, all the physical qubits within a single cell are forced to behave as a single virtual qubit. This process generates a two-dimensional $L \times L$ lattice with open boundary conditions of these virtual variables. Here, $L^{2}$ is the number of $\mathrm{K}_{4,4}$ cells on the Chimera graph with $N=8 L^{2}$ physical variables (qubits) and $L^{2}$ virtual variables.

2. The embedded instances are then generated on the virtual lattice with a given $\alpha$ and $R$.

These K-FCL problems [35] have considerably fewer (virtual) variables than other benchmarks, but have proven to be computationally difficult for many heuristics, in particular the HFS and PT+ICM solvers [21]. We emphasize, however, that the virtual problem is planar and can therefore be solved in polynomial time using minimumweight-perfect-matching techniques [36, 37]. As such, any speedup claims based on these problems have to be taken with a grain of salt.

\section{B. Deceptive cluster loop benchmark problems}

Inspired by the K-FCL benchmark problems, we have developed a new class of problems we call deceptive cluster loops. Although the ground state of the problem cannot be planted and therefore has to be computed with other efficient heuristics, we show that while the DW2000Q device maintains its performance for this class of problems, all other known heuristics struggle with solving these instances. In addition, the virtual problem cannot be easily decoded, i.e., the problems cannot be solved in polynomial time or with other clever approximations that exploit the logical structure [8].

The structure of the DCL problems can be summarized as follows: Starting from an embedded K-FCL instance, all the inter-cell couplers between $\mathrm{K}_{4,4}$ cells are multiplied by a factor $\lambda$, whereas all intra-cell couplers have magnitude 1 . To make the problem easier and allow for a better analysis of the computational scaling, we break the loops removing one of the ferromagnetic couplings.
One of the main feature of the proposed DCL problems that distinguishes them from other FCL-like models $[18,21,22]$ is the presence of two distinct limits for small and large $\lambda$. For small $\lambda$, i.e., in the limit of weak inter-cell couplings, each unit cell results to be strongly connected and therefore, behaves like a single virtual variable. In particular, when $\lambda \rightarrow 1$, the DCL problems are equivalent to K-FCL problems. The corresponding Ising model has a two-dimensional planar square lattice as the underlying graph and therefore can be solved in polynomial time [37]. On the other hand, in the limit of large $\lambda$, i.e., either horizontal or vertical chains that go across different unit cells become strongly coupled. Recalling that the Chimera graph is bipartite, there must exist a gauge transformation, i.e., a combination of spin flips in one of the two partitions, such that all the inter-cell couplings can be fixed to be ferromagnetic. Since in the limit of $\lambda \gg 1$ the energy contribution of intra-cell couplings is small compared to the energy contribution of inter-cell couplings, the ground state likely has all the chains polarized and therefore, they can be treated as single "virtual" variables and the corresponding virtual model is the fullyconnected bipartite model [38]. For intermediate values of $\lambda$, the DCL problems become a nontrivial combination of the two limits and therefore, optimal states cannot be mapped onto either virtual models. The effect becomes most pronounced when $\left|J_{i j}\right|$ for the inter-cell couplers is comparable to the connectivity of the intra-cell variables, i.e., $\left|J_{i j}\right| \approx 5-6$ for the current D-Wave Chimera architecture, where the local intra-cell environment felt by a variable in the $\mathrm{K}_{4,4}$ cell competes with the strength of the inter-cell couplers.

From a physical point of view, the DCL problems have another important property which makes them interesting in their own right: By continuously changing the scaling parameter $\lambda$, it is possible to modify the critical spin-glass temperature from $T_{c}=0(\lambda \sim 1)$ [12], to $T_{c}>0(\lambda \gg 1)$ [38]. Therefore, it would be interesting to understand the nature of the spin-glass phase for intermediate $\lambda$ where the system is neither planar or fully-connected [39].

\section{RESULTS}

In this Section, we compare the DW2000Q quantum chip against two of the fastest classical heuristics for Chimera Hamiltonians, namely the Hamze-de FreitasSelby (HFS) heuristic and the parallel tempering isoenergetic cluster method (PT+ICM) [40]. Both HFS and PT+ICM have been modified to correctly compute TTS as described in Eq. (3). Moreover, PT+ICM has been further optimized to exploit the knowledge of the virtual ground states in both limits of small $(\lambda \rightarrow 1)$ and large $(\lambda \gg 1)$ scaling (referred to as $\mathrm{PT}+\mathrm{ICM}+\mathrm{L}$ ). In particular, $\mathrm{TTS}_{\mathrm{PT}+\mathrm{ICM}+\mathrm{L}}$ is computed by running $\mathrm{PT}+\mathrm{ICM}$ from either an initial random state or from one of the two virtual ground states and then taking the minimum value. For each linear size $L$, we generated 100 DCL 

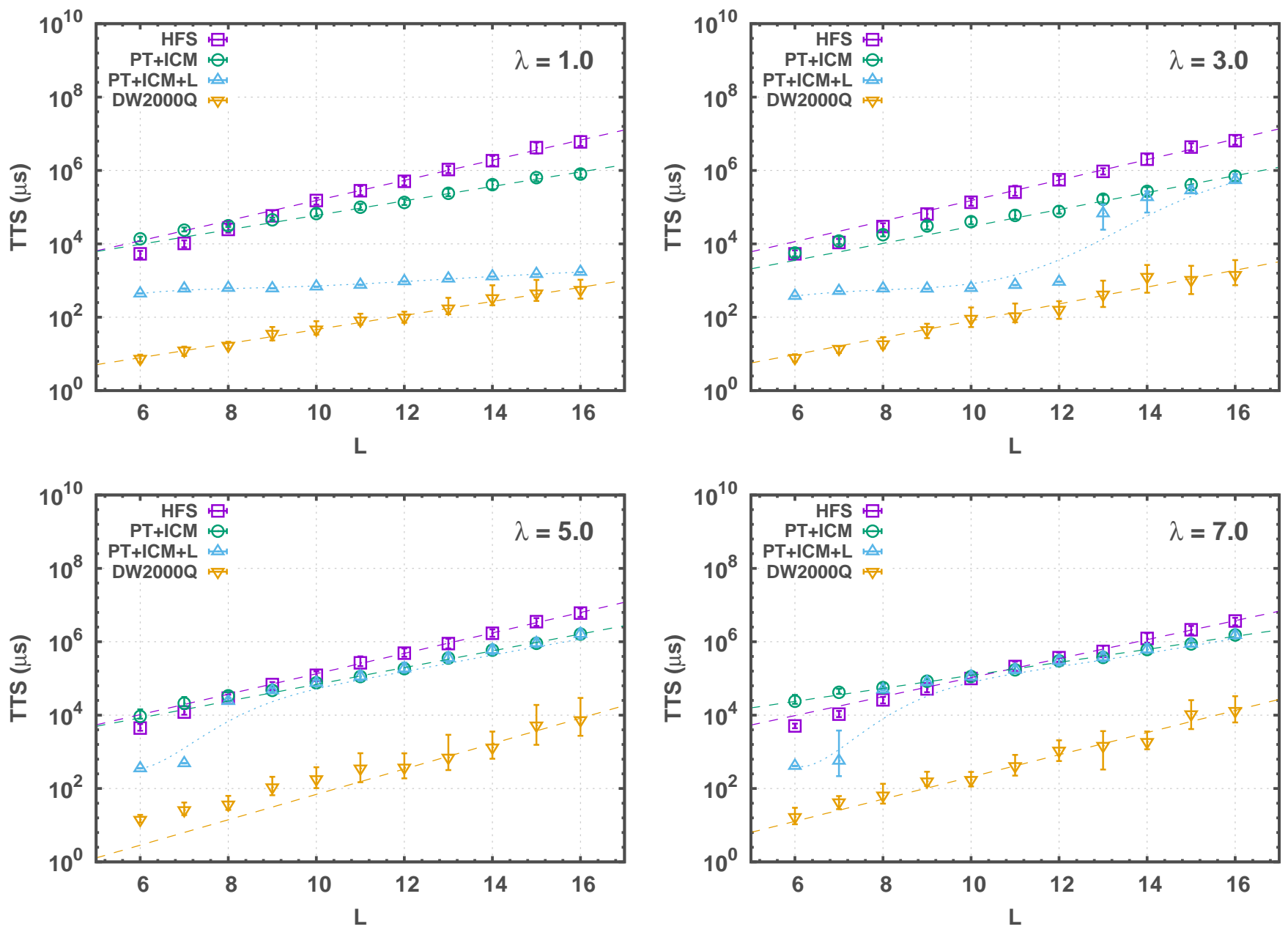

Figure 1. Time-to-solution (TTS) for the parallel tempering isoenergetic cluster method (PT+ICM), the Hamze-de Freitas-Selby (HFS) heuristic, as well for the D-Wave 2000Q (DW2000Q) quantum chip. All data points are for fixed scaling $\lambda$ while changing the linear problem size $L$. For this analysis, we also used a modified PT+ICM algorithm (PT+ICM+L) to take advantage of the knowledge of the virtual ground state for both small $(\lambda \rightarrow 1)$ and large $(\lambda \gg 1)$ scaling limits (see main text for more details). For DW2000Q, PT+ICM and HFS, fits are obtained by considering the last 5 points only (fit parameters are reported in the Appendix). For $\mathrm{PT}+\mathrm{ICM}+\mathrm{L}$, points are fit with a Bezier curve to guide the eye.

instances with parameters $\alpha=0.24$ and $R=1$ (instances at different $\lambda$ have been obtained by properly rescaling the inter-cell couplings). In all plots, points represent the median of the distribution while the error bar correspond to the $35 \%-65 \%$ percentiles. If not otherwise indicated, DW2000Q annealing time has been fixed to the minimum allowed, namely $5 \mu \mathrm{s}$. Simulation parameters for the classical heuristics are listed in the Appendix.

Figure (1) summarizes our results where DW2000Q is compared to both HFS and PT $+\mathrm{ICM}$. Interestingly, excluding the region of small $\lambda$ where $\mathrm{PT}+\mathrm{ICM}+\mathrm{L}$ is designed to be the fastest, DW2000Q always performs better than the two classical heuristics for the considered values of $\lambda$, being approximately $10^{2}$ times faster for $\lambda=7$. To better appreciate the different computational scaling among the classical and quantum heuristics we analyzed, Fig. (2), top panels, reports the scaling exponent $\beta$ of an exponential fit of the form:

$$
\log _{10} \operatorname{TTS}\left(t_{\text {run }}\right)=\beta t_{\text {run }}+\alpha .
$$

In the plots, boxes represent the confidence interval 35\%$65 \%$ for $\beta$ computed using only the $50 \%$ percentile of TTS while whisker bars represent the confidence interval $35 \%$ $65 \%$ for $\beta$ computed using the $35 \%-65 \%$ percentile of the TTS. As one can see, while HFS is statistically indistinguishable from the DW2000Q data, $\mathrm{PT}+\mathrm{ICM}$ performs slightly better for large $\lambda$. However, the better performance for $\mathrm{PT}+\mathrm{ICM}$ for large $\lambda$ can be explained by noticing that $\mathrm{PT}+\mathrm{ICM}$ has been optimized for each $\lambda$ while both DW2000Q and HFS use the same setup regardless of the value of $\lambda$. Figure (2, bottom panels, shows that DW2000Q is consistently faster than both HFS and $\mathrm{PT}+\mathrm{ICM}$ by, on average, a factor of $10^{2}$. Unfortunately, we cannot "certify" the DW2000Q computational scaling because we are not able to find the optimal annealing 



Figure 2. Exponential fit parameters for the time-to-solution (TTS) of the form $\log _{10}$ TTS $=\beta t_{\text {run }}+\alpha$, for DW2000Q , $\mathrm{PT}+\mathrm{ICM}$ and HFS. While the computational scaling parameters $\beta$ (top) are not significantly different, DW2000Q is over 100 times faster than PT+ICM and HFS when analyzing the prefactor $\alpha$ (bottom) . The linear regression is computed by considering only the last five linear sizes $L$ [see Fig. (9)].

time for the allowed minimum annealing time in the device. Still, as shown in Fig. (3), we have strong indication that the computational scaling we have found is reliable because of its stability for a large variation of annealing times [41].

To further analyze the effects of the rescaling factor $\lambda$ in Fig. (4) we show the performance of DW2000Q compared to $\mathrm{PT}+\mathrm{ICM}, \mathrm{PT}+\mathrm{ICM}+\mathrm{L}$, and HFS at fixed linear size of the system $L$. As expected, $\mathrm{PT}+\mathrm{ICM}+\mathrm{L}$ performs the best for both small and large $\lambda$, while its performance quickly degenerates for $\lambda \sim 7$, i.e., in the region where the true ground state is a nontrivial overlap of the virtual ground states at either small or large $\lambda$ [see Fig. (8) for the number of "broken" virtual variables for either the virtual planar model or the virtual fully-connected bipartite model]. In contrast, the DW2000Q performance gradually decreases by increasing the scaling factor $\lambda$ (precision issues $[13,38]$ may be one of the dominant

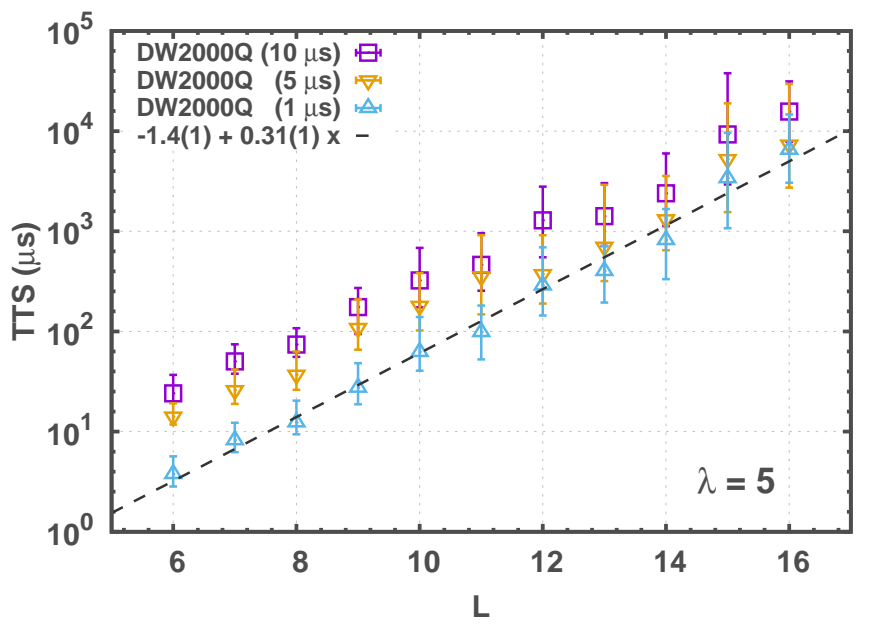

Figure 3. Comparison of the time-to-solution (TTS) for DW2000Q by varying annealing time. Despite the fact that we are not able to identify an optimal annealing time, the last five linear sizes $L$ are consistent for a wide range of annealing times.

factor of the loss of performance).

As final remark, it is important to stress that the advantage is not just on the typical instance. Indeed, as it is shown in Fig. (5), DW2000Q performs best even in an instance by instance comparison, when contrasted to HFS and PT+ICM.

\section{ANALYSIS OF THE INITIALIZATION AND READOUT TIME}

While the manufacturing of quantum hardware is becoming more advanced with better superconducting circuits and more resilient qubits, quantum technology, in general, is still in its infancy. Indeed, technological problems such as long initialization and readout times are still a burden for any early quantum device. Hopefully, this problem will be resolved in the near future with, e.g., cryogenic control electronics. In particular, the DW2000Q quantum chip needs $t_{\text {read }}=123 \mu s$ to read a configuration at the end of the annealing process. Moreover, the DW2000Q quantum chip requires $t_{\mathrm{ini}}=21 \mu \mathrm{s}$ between anneals to reduce memory effects. In many cases, the total amount of $t_{\text {ini }}+t_{\text {read }}=144 \mu s$ is an order of magnitude larger than the optimal annealing time. Consequently, the inclusion of the initialization/readout time affects more optimization runs that involve fast anneals with many repetitions rather than slow anneals with few repetitions.

As described in Sec. IV, the optimal annealing time for the DCL class of problems is $1 \mu s$ (see Fig. 6, left panel). Therefore, the addition of $144 \mu s$ drastically reduces the performance of the DW2000Q quantum chip hence limiting the quantum advantage shown in Fig. 4. The optimization described in Sec. IV does not take into 


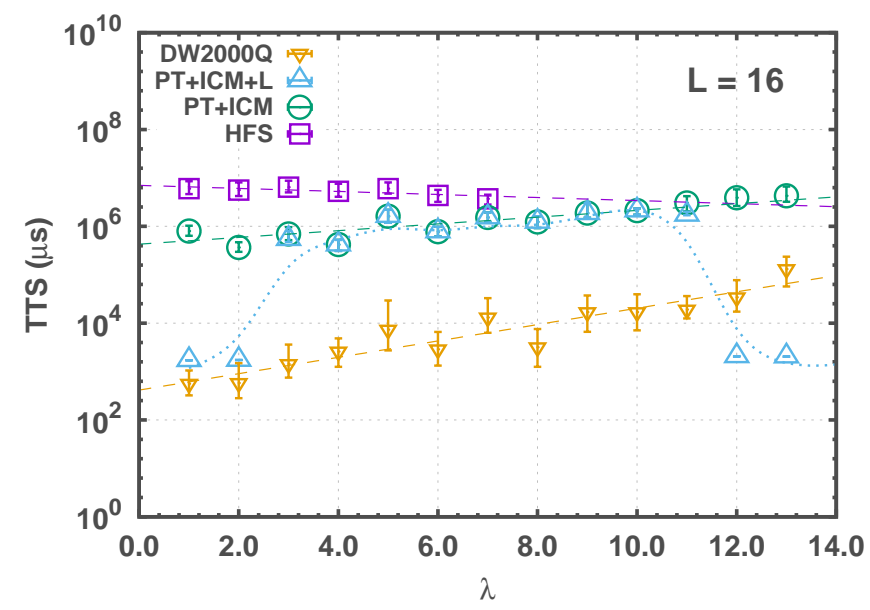

Figure 4. Time-to-solution (TTS) for the parallel tempering isoenergetic cluster method $(\mathrm{PT}+\mathrm{ICM})$, the Hamzede Freitas-Selby (HFS) heuristic, as well for D-Wave 2000Q (DW2000Q) quantum chip. All data points are for fixed linear lattice size $L=16$. For this analysis, we also used a modified $\mathrm{PT}+\mathrm{ICM}$ algorithm $(\mathrm{PT}+\mathrm{ICM}+\mathrm{L})$ to take advantage of the knowledge of the virtual grounds state for both small $(\lambda \rightarrow 1)$ and large $(\lambda \gg 1)$ scaling limits (see main text for more details). Data show that HFS is barely affected by the scaling $\lambda$, while the DW2000Q performance slowly degrades by increasing $\lambda$ (most likely due to precision issues $[13,38]$ ). As expected, PT $+\mathrm{ICM}+\mathrm{L}$ performs the best for both small and large $\lambda$, while its performance quickly degenerates for $\lambda \sim 7$, i.e., in the region where the true ground state is different from the virtual ground state. Interestingly, for $\lambda \sim 7$, DW2000Q has the better performance resulting and is at least 100 times faster than HFS and PT+ICM.

account the initialization and readout times. A more careful analysis shows that the inclusion of the initialization and readout times shifts the optimal annealing time to larger values (see Fig. 6, right panel). Indeed, once the initialization and readout times are included in the optimization, the overall performance is improved by having slower anneals with fewer repetitions. Interestingly, as shown in Fig. 7, the overall slowdown due to the introduction of the initialization and readout times can be reduce by a factor $\sim 140$ to a mere factor $\sim 30$, which allows the DW2000Q device to keep a small computational advantage in comparison to the HFS and PT+ICM heuristics.

\section{INCLUSION OF POWER CONSUMPTION}

While most of the benchmark studies have largely focused on pure computational speed, the inclusion of power consumption has been largely neglected in the literature [42]. With ever-growing data centers, power consumption has become an important issue and "greener" computational solutions are highly sought after.

A large-sized data center like the one hosted at NASA Ames [43, 44] has a typical energy consumption of approximately $5 \mathrm{MW}$, with a $4: 1$ ratio between power usage and cooling (during benchmarking). With more than 245000 cores for the current NASA high-performance computing cluster, the typical energy consumption is approximately $20 \mathrm{~W} /$ core. In contrast, the energy consumption of the DW2000Q quantum processing unit is approximately $150 \mathrm{pW}$. Keeping the quantum processing unit cooled to $20 \mathrm{mK}$ requires approximately $15-25 \mathrm{~kW}$. In our analysis, we found that the DW2000Q device was approximately $10^{2}-10^{3}$ times faster than the used PT+ICM and HFS heuristics. Therefore, to compete against DW2000Q, $\sim 10^{2}-10^{3}$ compute cores are needed running in parallel with a total energy consumption between 2 and $20 \mathrm{~kW}$. Therefore, power consumption is, overall, comparable. However, there is a remarkable difference: The data center uses $80 \%$ of the total consumed energy to run the computers, while the DW2000Q device requires only $10^{-14 \%}$ of the power to run the quantum processing unit. Therefore, while an improvement of the power usage effectiveness (PUE) [45, 46] for the classical data center would eventually reduce the total cooling power of $20 \%$, far more efficient cooling alternatives are needed to reduce the quantum PUE (qPUE). It is unclear how the qPUE can be reduced due to the cryogenic requirements for quantum processing units. However, dry dilution refrigerators with more efficient pumping systems might improve this metric [47].

\section{CONCLUSIONS}

In conclusion, we present the first class of tunable benchmark problems - Deceptive Cluster Loops (DCL) for which the D-Wave quantum chip (DW2000Q) shows an advantage over the currently best classical heuristics, namely the parallel tempering isoenergetic cluster method $(\mathrm{PT}+\mathrm{ICM})$ and the Hamze-de Freitas-Selby (HFS) algorithm. The benchmarks are characterized by a control parameter $\lambda$, the scaling factor of the inter-cell couplings, that allows to continuously transform the model from a virtual planar model $(\lambda \sim 1)$ to a virtual fully-connected bipartite problem $(\lambda \gg 1)$. While classical heuristics are faster in the small- and large- $\lambda$ limit where the logical structure can be exploited, DW2000Q is the fastest in the crossover region $\lambda \sim 7$, where the DCL problems are neither virtual planar nor virtual fully-connected bipartite. Indeed, while the computational scaling is comparable among classical and quantum heuristics, the DW2000Q device is approximately two orders of magnitude faster than the currently best known heuristics (PT $+\mathrm{ICM}$ and HFS) with a comparable scaling. This result represents the first of its kind since the inception of the D-Wave quantum chip. We also show that the inclusion of the initialization and readout times may reduce the performance of the DW2000Q quantum chip. However, we also show that a proper optimization of the time to solution mitigates the effects of the inclusion of the initialization and readout times, allowing the DW2000Q quantum chip to have a small advantage over 

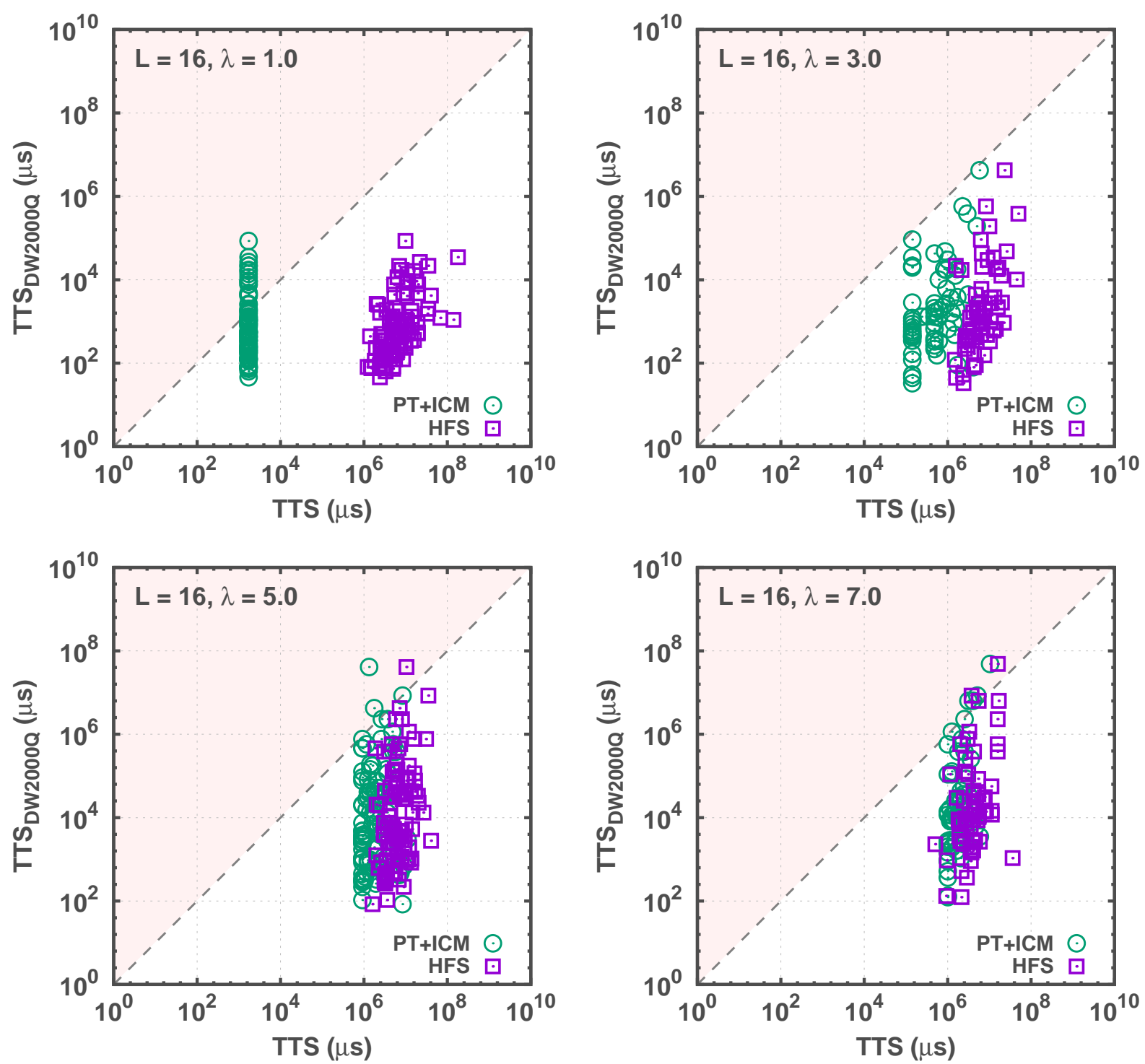

Figure 5. Instance by instance comparison of the Time-to-solution (TTS) between the different heuristics used, the parallel tempering isoenergetic cluster method (PT+ICM ) and the Hamze-de Freitas-Selby (HFS ) algorithms, and the D-Wave 2000Q (DW2000Q) quantum chip. As one can see, DW2000Q is consistently faster than HFS and PT+ICM.


Figure 6. Time to solution (TTS) by varying the annealing time $T_{\text {ann }}$ without (left panel) and with (right panel) the inclusion of the initialization and readout time. Interestingly, the inclusion of the initialization and readout time moves the optimal annealing time to larger values. 


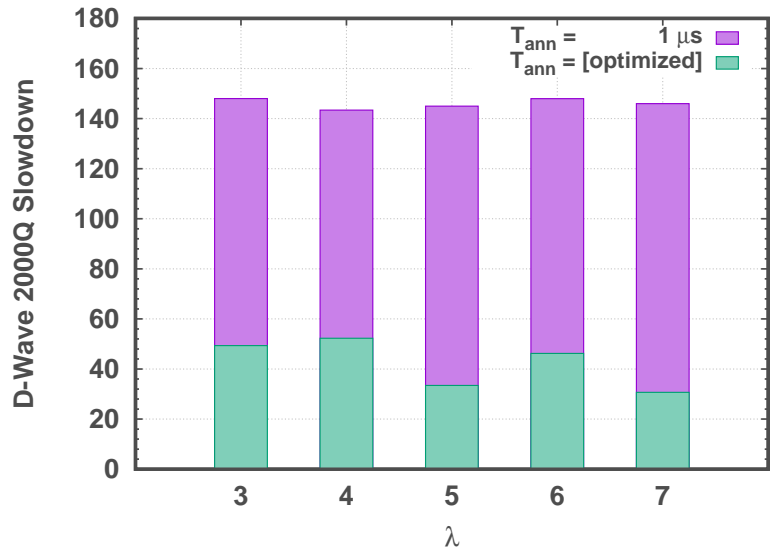

Figure 7. Ratio between the time to solution (TTS) before and after the inclusion of the initialization and readout time (slowdown) for $L=16$. As one can see, the optimization of TTS with the inclusion of the initialization and readout time drastically reduces the slowdown of the DW2000Q.

the best classical heuristics to date.

\section{ACKNOWLEDGMENTS}

H. G. K. acknowledges support from the National Science Foundation (Grant No. DMR-1151387) and thanks M. Thom for multiple discussions on power consumption of the DW2000Q devices. He also thanks N. Artner for support. S. M. acknowledges E. G. Rieffel for the careful reading of the manuscript and useful discussion, C. E. Henze and the NASA Ames Research Center for support and computational resources. We would like to thank M. Steininger and J. King for feedback on the manuscript. This research is based upon work supported by the Office of the Director of National Intelligence (ODNI), Intelligence Advanced Research Projects Activity (IARPA), via Interagency Umbrella Agreement IA1-1198. The views and conclusions contained herein are those of the authors and should not be interpreted as necessarily representing the official policies or endorsements, either expressed or implied, of the ODNI, IARPA, or the U.S. Government. The U.S. Government is authorized to reproduce and distribute reprints for Governmental purposes notwithstanding any copyright annotation thereon.

\section{Appendix A: Number of Broken Virtual Variables in the DCL Model}

Our numerical simulations for $L=16$ suggest that the DCL model reduces to the virtual planar model for $\lambda \lesssim 2$, while it is a virtual fully-connected bipartite for $\lambda \gtrsim 10$ [see Fig. (4)]. Figure (8) shows the number of "broken" virtual variables for either the virtual planar model or the virtual fully-connected bipartite model. The interesting regime is obtained when $\lambda \sim 7$, i.e., when the DCL model is neither a virtual planar model nor a virtual fully-connected bipartite model. In this regime, the ground state of the DCL model cannot be found by solving a corresponding virtual problems and therefore, logical structures cannot be exploited as in Refs. [18, 21, 37].

\section{Appendix B: Simulation parameters}

In this Section, we briefly report the main parameters we used for our experiments and numerical simulations.

$D C L$ Random Instances - We randomly generate 100 instances for each system size $(L=6, \ldots, 16)$. The instances are generated by following the prescription in Ref. [21] (with $\alpha=0.24, R=\rho=1$ ) and then properly rescaling the inter-cell couplings by a factor $\lambda$. To make the problem easier and allow for a better analysis of the computational scaling, we break the loops removing one of the ferromagnetic couplings. For consistency, the same instances have been used for all values of $\lambda$. Unlike in Ref. [21], we used all available qubits, i.e., some of the unit cells are not complete.

DW2000Q Parameters - For all experiments, we use the minimum allowed annealing time of $5 \mu \mathrm{s}$ and 100 gauges $\times 1000$ runs, i.e., 100000 total readouts. The initialization time and the readout time have not been included in the calculation of the TTS.

PT + ICM Parameters - The lowest and highest temperature for parallel tempering have been chosen to be 1 and 10, respectively, to maximize the performance of PT+ICM. Optimal sweeps for each instance and $\lambda$ have been determined by computing the cumulative distribution of the probability to find the ground state (100 runs for each instance and $\lambda$ ). The overall optimal number of sweeps is then obtained by bootstrapping the optimal number of sweeps for each instance. For all simulations, the number of sweeps has been optimized to minimize the TTS of the $50 \%$ percentile. The initialization time and the readout time have been not included in the calculation of the TTS.

$P T+I C M+L$ Parameters - The parameters used are the same as for PT+ICM. The computational time to find the ground state of either the virtual planar model of the virtual fully-connected bipartite model has been set to zero (in reality, the computational time to find the ground state of the fully-connected bipartite model is nonnegligible).

HFS Parameters - The option -S13, namely "Exhaust maximal tree width 1 subgraphs" with partial random state initialization, has been used. Optimal sweeps for each instance and $\lambda$ have been determined by computing the cumulative distribution of the probability to find the ground state (100 runs for each instance and $\lambda$ ). 
Planar model

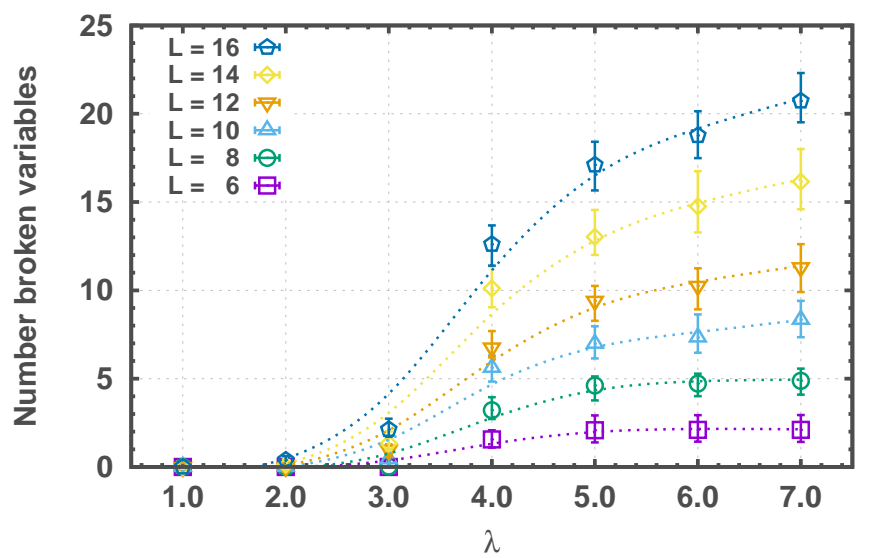

FCB model

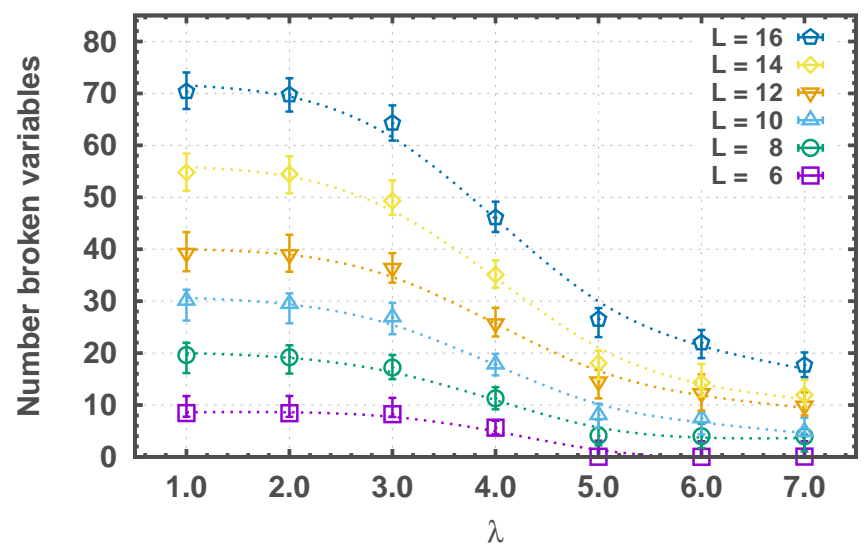

Figure 8. (Left panel) Number of broken virtual variables with respect to the virtual planar model (limit for $\lambda \rightarrow 1$ ). (Right panel) Number of broken virtual variables with respect to the virtual fully-connected bipartite model (limit for $\lambda \gg 1$ ). As one can see, the number of broken variables goes to zero when the scaling $\lambda$ approaches the limit of the corresponding virtual model.

The overall optimal number of sweeps is then obtained by bootstrapping the optimal number of sweeps for each instance. For all the simulations, the number of sweeps has been optimized to minimize the TTS of the $50 \%$ percentile. The initialization time and the readout time have been not included in the calculation of the TTS.

[1] T. Kadowaki and H. Nishimori, Quantum annealing in the transverse Ising model, Phys. Rev. E 58, 5355 (1998).

[2] E. Farhi, J. Goldstone, S. Gutmann, J. Lapan, A. Lundgren, and D. Preda, A quantum adiabatic evolution algorithm applied to random instances of an NP-complete problem, Science 292, 472 (2001).

[3] A. B. Finnila, M. A. Gomez, C. Sebenik, C. Stenson, and J. D. Doll, Quantum annealing: A new method for minimizing multidimensional functions, Chem. Phys. Lett. 219, 343 (1994).

[4] R. Martoňák, G. E. Santoro, and E. Tosatti, Quantum annealing by the path-integral Monte Carlo method: The two-dimensional random Ising model, Phys. Rev. B 66, 094203 (2002).

[5] G. Santoro, E. Martoňák, R. Tosatti, and R. Car, Theory of quantum annealing of an Ising spin glass, Science 295, 2427 (2002).

[6] A. Das and B. K. Chakrabarti, Quantum Annealing and Analog Quantum Computation, Rev. Mod. Phys. 80, 1061 (2008).

[7] T. F. Rønnow, Z. Wang, J. Job, S. Boixo, S. V. Isakov, D. Wecker, J. M. Martinis, D. A. Lidar, and M. Troyer, Defining and detecting quantum speedup, Science 345, 420 (2014).

[8] S. Mandrà, Z. Zhu, W. Wang, A. Perdomo-Ortiz, and H. G. Katzgraber, Strengths and weaknesses of weakstrong cluster problems: A detailed overview of state-ofthe-art classical heuristics versus quantum approaches,
Exponential Fits - For all the linear regressions, only the last 5 system sizes $(L=12, \ldots, 16)$ have been used. $\log _{10}$ TTS fits for the $50 \%$ percentile have been obtained using a linear least squares model. $\log _{10}$ TTS fits for the $35 \%-65 \%$ confidence have been computed by randomly extract values in the confidence interval, one for each size, and then bootstrapping the linear regression data. Figure (9) shows the fits for different values of $\lambda$.
Phys. Rev. A 94, 022337 (2016).

[9] M. W. Johnson, M. H. S. Amin, S. Gildert, T. Lanting, F. Hamze, N. Dickson, R. Harris, A. J. Berkley, J. Johansson, P. Bunyk, et al., Quantum annealing with manufactured spins, Nature 473, 194 (2011).

[10] N. G. Dickson, M. W. Johnson, M. H. Amin, R. Harris, F. Altomare, A. J. Berkley, P. Bunyk, J. Cai, E. M. Chapple, P. Chavez, et al., Thermally assisted quantum annealing of a 16-qubit problem, Nat. Commun. 4, 1903 (2013).

[11] S. Boixo, T. F. Rønnow, S. V. Isakov, Z. Wang, D. Wecker, D. A. Lidar, J. M. Martinis, and M. Troyer, Evidence for quantum annealing with more than one hundred qubits, Nat. Phys. 10, 218 (2014).

[12] H. G. Katzgraber, F. Hamze, and R. S. Andrist, Glassy Chimeras Could Be Blind to Quantum Speedup: Designing Better Benchmarks for Quantum Annealing Machines, Phys. Rev. X 4, 021008 (2014).

[13] H. G. Katzgraber, F. Hamze, Z. Zhu, A. J. Ochoa, and H. Munoz-Bauza, Seeking Quantum Speedup Through Spin Glasses: The Good, the Bad, and the Ugly, Phys. Rev. X 5, 031026 (2015).

[14] B. Heim, T. F. Rønnow, S. V. Isakov, and M. Troyer, Quantum versus classical annealing of Ising spin glasses, Science 348, 215 (2015).

[15] I. Hen, J. Job, T. Albash, T. F. Rønnow, M. Troyer, and D. A. Lidar, Probing for quantum speedup in spin-glass problems with planted solutions, Phys. Rev. A 92, 042325 

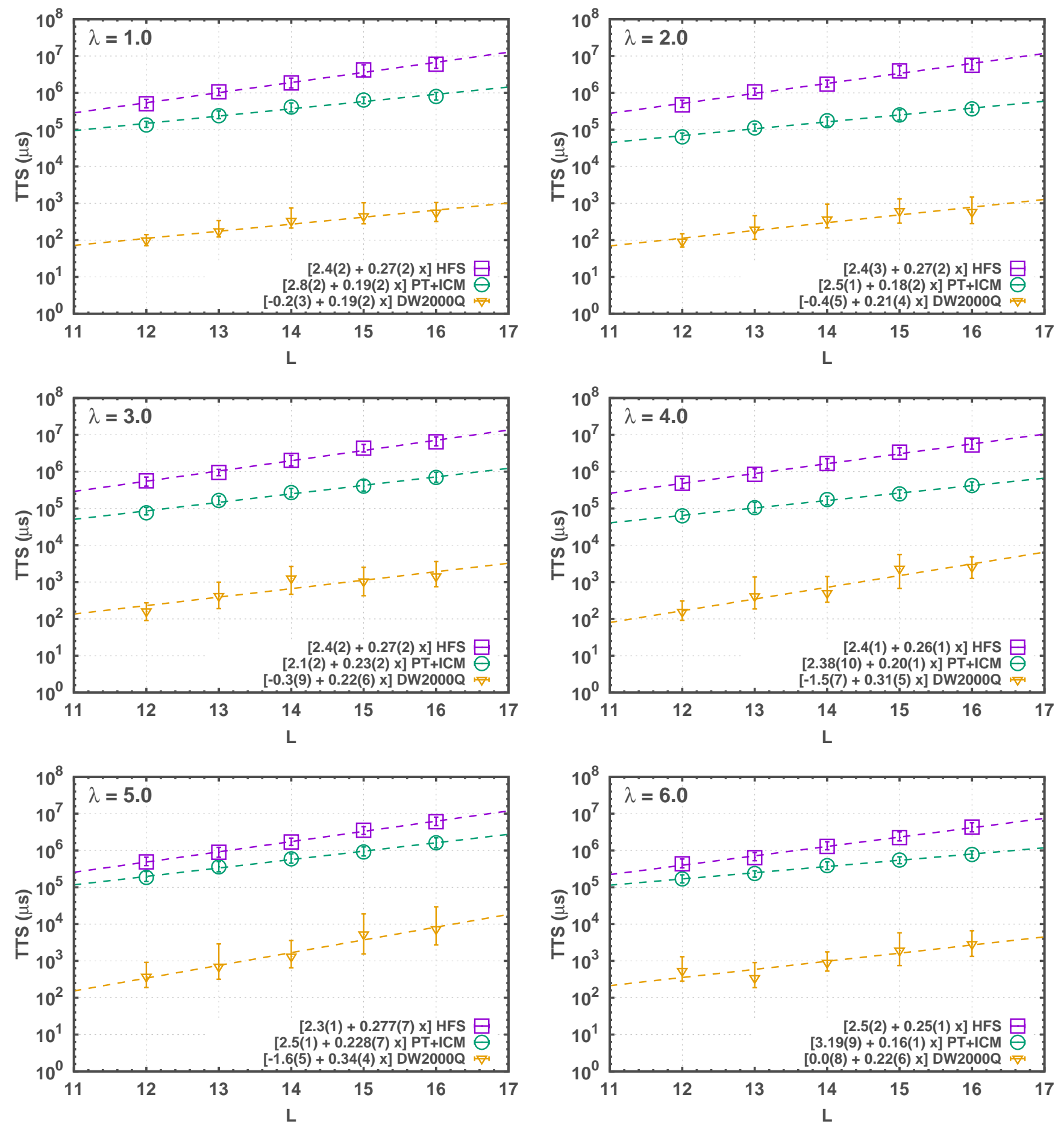

Figure 9. Linear regressions by varying the linear system size $L$, at different inter-cell couplings scaling $\lambda$.

(2015).

[16] T. Albash, T. F. Rønnow, M. Troyer, and D. A. Lidar, Reexamining classical and quantum models for the DWave One processor, Eur. Phys. J. Spec. Top. 224, 111 (2015).

[17] V. Martin-Mayor and I. Hen, Unraveling Quantum Annealers using Classical Hardness, Nature Scientific Re- ports 5, 15324 (2015).

[18] A. D. King, T. Lanting, and R. Harris, Performance of a quantum annealer on range-limited constraint satisfaction problems (2015), arXiv:1502.02098.

[19] J. Marshall, V. Martin-Mayor, and I. Hen, Practical engineering of hard spin-glass instances, Phys. Rev. A 94, 012320 (2016). 
[20] V. S. Denchev, S. Boixo, S. V. Isakov, N. Ding, R. Babbush, V. Smelyanskiy, J. Martinis, and H. Neven, What is the Computational Value of Finite Range Tunneling?, Phys. Rev. X 6, 031015 (2016).

[21] J. King, S. Yarkoni, J. Raymond, I. Ozfidan, A. D. King, M. M. Nevisi, J. P. Hilton, and C. C. McGeoch, Quantum Annealing amid Local Ruggedness and Global Frustration (2017), (arXiv:quant-phys/1701.04579).

[22] T. Albash and D. A. Lidar, Evidence for a Limited Quantum Speedup on a Quantum Annealer (2017), (arXiv:quant-phys/1705.07452).

[23] P. Bunyk, E. Hoskinson, M. W. Johnson, E. Tolkacheva, F. Altomare, A. J. Berkley, R. Harris, J. P. Hilton, T. Lanting, and J. Whittaker, Architectural Considerations in the Design of a Superconducting Quantum Annealing Processor, IEEE Trans. Appl. Supercond. 24, 1 (2014).

[24] F. Hamze and N. de Freitas, in Proceedings of the 20th Conference on Uncertainty in Artificial Intelligence (AUAI Press, Arlington, Virginia, United States, 2004), UAI '04, p. 243, ISBN 0-9749039-0-6.

[25] A. Selby, Efficient subgraph-based sampling of Isingtype models with frustration (2014), (arXiv:condmat/1409.3934).

[26] Z. Zhu, A. J. Ochoa, and H. G. Katzgraber, Efficient Cluster Algorithm for Spin Glasses in Any Space Dimension, Phys. Rev. Lett. 115, 077201 (2015).

[27] S. Morita and H. Nishimori, Mathematical Foundation of Quantum Annealing, J. Math. Phys. 49, 125210 (2008).

[28] G. E. Santoro and E. Tosatti, TOPICAL REVIEW: Optimization using quantum mechanics: quantum annealing through adiabatic evolution, J. Phys. A 39, R393 (2006).

[29] M. H. S. Amin, D. V. Averin, and J. A. Nesteroff, Decoherence in adiabatic quantum computation, Phys. Rev. A 79, 022107 (2009).

[30] T. Albash and D. A. Lidar, Decoherence in adiabatic quantum computation, Phys. Rev. A 91, 062320 (2015).

[31] W. Wang, J. Machta, and H. G. Katzgraber, Bond chaos in spin glasses revealed through thermal boundary conditions, Phys. Rev. B 93, 224414 (2016).

[32] K. Nishimura, H. Nishimori, A. J. Ochoa, and H. G. Katzgraber, Retrieving the ground state of spin glasses using thermal noise: Performance of quantum annealing at finite temperatures, Phys. Rev. E 94, 032105 (2016).

[33] J. Marshall, E. G. Rieffel, and I. Hen, Thermalization, freeze-out and noise: deciphering experimental quantum annealers, Phys. Rev. App. 6, 064025 (2017).

[34] S. Mandrà, G. G. Guerreschi, and A. Aspuru-Guzik, Adiabatic quantum optimization in the presence of discrete noise: Reducing the problem dimensionality, Phys. Rev. A 92, 062320 (2015).

[35] It has been tempting to refer to the approach developed in Ref. [21] "frustrated cluster loops Hen à la King," but that would be a mouthful every time it appears in the text.

[36] V. Kolmogorov, Blossom V: A new implementation of a minimum cost perfect matching algorithm, Math. Prog. Comp. 1, 43 (2009).

[37] S. Mandrà and H. G. Katzgraber, The pitfalls of planar spin-glass benchmarks: Raising the bar for quantum annealers (again), Quantum Sci. Technol. 2, 038501 (2017).

[38] D. Venturelli, S. Mandrà, S. Knysh, B. O'Gorman, R. Biswas, and V. Smelyanskiy, Quantum Optimization of Fully Connected Spin Glasses, Phys. Rev. X 5, 031040 (2015).

[39] S. Mandrà and H. G. Katzgraber, In preparation (2018).

[40] An analysis using SQA has not been included in this study because preliminary results on the instances studied in Ref. [22] show that PT+ICM is at least one order of magnitude faster than SQA with a comparable scaling.

[41] Experiments for $1 \mu \mathrm{s}$ have been obtained using the DW2000Q quantum chip recently installed at NASA Ames.

[42] To our knowledge, the only discussion of power consumption between quantum and classical hardware can be found in a whitepaper published on D-Wave Systems Inc.'s website [https://goo.gl/v72k2f].

[43] (2016), top500 List - June 2016, URL https://www. top500.org/list/2016/06/.

[44] Private communication with Christopher E. Henze (NASA Ames).

[45] G. A. Brady, N. Kapur, J. L. Summers, and H. M. Thompson, A case study and critical assessment in calculating power usage effectiveness for a data centre, Energy conversion and management 76, 155 (2013).

[46] The Green Grid, URL http://www . thegreengrid.org.

[47] We note that a comprehensive analysis of the power consumption requires the inclusion of embedding, programming, and readout times, as well as considerations on dissipated energy, for both classical and quantum machines. 